PROCEEDINGS OF THE

AMERICAN MATHEMATICAL SOCIETY

Volume 132, Number 5, Pages 1549-1558

S 0002-9939(03)07328-3

Article electronically published on December 19, 2003

\title{
CONTACT 3-MANIFOLDS WITH INFINITELY MANY STEIN FILLINGS
}

\author{
BURAK OZBAGCI AND ANDRÁS I. STIPSICZ
}

(Communicated by Ronald A. Fintushel)

\begin{abstract}
Infinitely many contact 3-manifolds each admitting infinitely many pairwise non-diffeomorphic Stein fillings are constructed. We use Lefschetz fibrations in our constructions and compute their first homologies to distinguish the fillings.
\end{abstract}

\section{INTRODUCTION}

A complex surface $V$ is Stein if it admits a proper holomorphic embedding $f: V \rightarrow \mathbb{C}^{n}$ for some $n$. For a generic point $p \in \mathbb{C}^{n}$, consider the map $\varphi: V \rightarrow \mathbb{R}$ defined by $\varphi(z)=\|z-p\|^{2}$. For a regular value $a \in \mathbb{R}$, the level set $M=\varphi^{-1}(a)$ is a smooth 3-manifold (oriented as $\partial \varphi^{-1}([0, a])$ ) with a distinguished 2-plane field $\xi=T M \cap i T M \subset T V$. It turns out that $\xi$ defines a contact structure on $M$ (for more about contact structures, see [1], 6]), and $S=\varphi^{-1}([0, a])$ is called a Stein filling of $(M, \xi)$. Topological properties of Stein fillings (and slightly more generally, of strong symplectic fillings) are in the focus of current research. Based on work of Eliashberg [4, McDuff [15] and Lisca [13], we know, for example, that for the lens space $L(p, q)$ equipped with a specific contact structure $\xi_{(p, q)}$ there is a finite list $\left\{S_{p, q}(n) \mid n=1, \ldots, n_{p, q}\right\}$ of 4 -manifolds such that any Stein filling of $\left(L(p, q), \xi_{(p, q)}\right)$ is diffeomorphic to some $S_{p, q}(n)$. Similar finiteness results have been verified for simple and simple elliptic singularities [16], [17], and for homeomorphism types of Stein fillings of the 3-torus $T^{3}[20$. Based on these examples, it was anticipated that a contact 3 -manifold $(M, \xi)$ admits only finitely many nondiffeomorphic Stein fillings. Genus-3 fibrations found by I. Smith [19] indicated that such an expectation is too ambitious in general.

In the following we show the existence of an infinite family of contact 3-manifolds each admitting infinitely many non-diffeomorphic Stein fillings.

Theorem 1.1. For all $g \geq 2$ there is a contact 3 -manifold $\left(M_{g}, \xi_{g}\right)$ such that $M_{g}$ is diffeomorphic to $M_{g^{\prime}}$ iff $g=g^{\prime}$ and each $\left(M_{g}, \xi_{g}\right)$ admits infinitely many pairwise non-diffeomorphic Stein fillings.

In fact, $M_{g}$ can be given as the boundary of the plumbing of the disk bundle over a genus- $g$ surface with Euler number zero and the disk bundle over a sphere with Euler number 2. In the proof of Theorem 1.1 we use Lefschetz fibrations to construct the Stein fillings, and we compute their first homology groups to distinguish

Received by the editors April 15, 2002.

2000 Mathematics Subject Classification. Primary 57R57, 57R17.

(C)2003 American Mathematical Society 
them. It would be most desirable to find infinitely many distinct fillings with trivial fundamental group; we hope to return to this point later. Regarding finiteness of Stein fillings, we conjecture the following:

Conjecture 1.2. Let $\chi(X)$ denote the Euler characteristic of the compact manifold $X$. Then the set $\mathcal{C}_{(M, \xi)}=\{\chi(S) \mid S$ is a Stein filling of $(M, \xi)\}$ is finite.

\section{Fiber sums of Lefschetz fibrations}

Let $\Sigma$ be a compact, oriented and connected surface of genus $g$ with $r$ marked points and $k$ boundary components. The mapping class group $\Gamma_{g, r}^{k}$ of $\Sigma$ consists of the isotopy classes of orientation-preserving self-diffeomorphisms of $\Sigma$ that are the identity on each boundary component and preserve the set of marked points. (The groups $\Gamma_{g, r}^{0}, \Gamma_{g, 0}^{k}$ and $\Gamma_{g, 0}^{0}$ will be abbreviated by $\Gamma_{g, r}, \Gamma_{g}^{k}$ and $\Gamma_{g}$, respectively.) We say that a $\Sigma$-bundle $P$ over $S^{1}$ has monodromy $h \in \Gamma_{g, r}^{k}$ iff $P$ is diffeomorphic to $(\Sigma \times I) /(h(x), 0) \sim(x, 1)$.

Definition 2.1. Let $X$ be a compact, connected, oriented, smooth four-manifold. A Lefschetz fibration on $X$ is a smooth map $\pi: X \rightarrow B$, where $B$ is a compact, connected, oriented surface, $\pi^{-1}(\partial B)=\partial X$, and furthermore each critical point of $\pi$ lies in int $X$ and has an orientation-preserving local coordinate chart on which $\pi\left(z_{1}, z_{2}\right)=z_{1}^{2}+z_{2}^{2}$.

It follows that $\pi$ has only finitely many critical points, and removing the corresponding singular fibers turns a Lefschetz fibration into a fiber bundle with a connected base space. Consequently, all but finitely many fibers of a Lefschetz fibration are smooth, compact and oriented surfaces, all having the same diffeomorphism type of a closed genus- $g$ surface for some $g$. We will assume that there is at most one critical point on each fiber. A Lefschetz fibration is called relatively minimal if no fiber contains an embedded 2-sphere of self-intersection number -1. Each critical point of a Lefschetz fibration corresponds to an embedded circle in a nearby regular fiber called a vanishing cycle, and the singular fiber is obtained by collapsing the vanishing cycle to a point. The boundary of a regular neighborhood of a singular fiber is a surface bundle over the circle. In fact, a singular fiber can be described by the monodromy of this surface bundle, which turns out to be a right-handed Dehn twist along the corresponding vanishing cycle. Once we fix an identification of $\Sigma$ with the fiber over a base point of $B$, the topology of the Lefschetz fibration is determined by its monodromy representation $\Psi: \pi_{1}(B-\{$ critical values $\}) \rightarrow \Gamma_{g}$. In case $B=D^{2}$ the monodromy along $\partial D^{2}=S^{1}$ is called the total monodromy of the fibration; according to the above, it is the product of right-handed Dehn twists corresponding to the singular fibers. A Lefschetz fibration over $S^{2}$ can be decomposed into two Lefschetz fibrations over $D^{2}$, one of which is trivial; consequently, a Lefschetz fibration over $S^{2}$ is determined by a relator in the mapping class group. Conversely, given a product of right-handed Dehn twists in the mapping class group, we can construct the corresponding Lefschetz fibration over $D^{2}$, and if the given product of right-handed Dehn twists is isotopic to the identity (and $g \geq 2$ ), then the fibration extends uniquely over $S^{2}$. The monodromy representation also provides a handlebody decomposition of a Lefschetz fibration over $D^{2}$ : we attach 2-handles to $\Sigma \times D^{2}$ along the vanishing cycles with framing -1 relative to the framing that the circle inherits from the fiber. (For a more detailed introduction to the theory of Lefschetz fibration, see [10].) 
Let $X \rightarrow S^{2}$ be a Lefschetz fibration with generic fiber $\Sigma$ and let $\gamma_{1}, \gamma_{2}, \cdots, \gamma_{s}$ denote the vanishing cycles of this fibration. Assume that $X \rightarrow S^{2}$ admits a section, i.e., there is $\sigma: S^{2} \rightarrow X$ with $\pi \circ \sigma=\mathrm{id}_{S^{2}}$. The following results are standard.

Lemma 2.2. The first homology group $H_{1}(X ; \mathbb{Z})$ is the quotient of $H_{1}(\Sigma ; \mathbb{Z})$ by the subgroup generated by the homology classes of the vanishing cycles.

Lemma 2.3. Let $X \sharp_{f} X$ denote the fiber sum of $X$ with itself by a self-diffeomorphism $f$ of the generic fiber $\Sigma$. Then $X \sharp_{f} X$ is a Lefschetz fibration with vanishing cycles $\gamma_{1}, \gamma_{2}, \cdots, \gamma_{s}, f\left(\gamma_{1}\right), f\left(\gamma_{2}\right), \cdots, f\left(\gamma_{s}\right)$.

Remark 2.4. If $\alpha$ is a simple closed curve on $\Sigma, t_{\alpha}$ is the corresponding Dehn twist and $f$ is an orientation-preserving self-diffeomorphism of $\Sigma$, then $f t_{\alpha} f^{-1}=t_{f(\alpha)}$ in $\Gamma_{g, r}^{k}$.

After this short introduction we begin our construction with a description of a set of words in the mapping class groups which was discovered by Korkmaz [12]. We focus on the odd genus case; for $g$ even see Remark 2.8. For $g=2 r+1 \geq 3$, the following relation holds in $\Gamma_{g}$ :

$$
W_{g}=\left(t_{B_{0}} t_{B_{1}} t_{B_{2}} \cdots t_{B_{g}} t_{a}^{2} t_{b}^{2}\right)^{2}=1,
$$

where $B_{0}, B_{1}, \cdots, B_{g}$ are shown in Figure 1 and $a$ and $b$ in Figure 4 Let $X_{g} \rightarrow$ $S^{2}$ denote the Lefschetz fibration corresponding to the relator $W_{g}$ in $\Gamma_{g}$. Now consider the fiber sum of $X_{g}$ with itself using the diffeomorphism $t_{a_{1}}^{n}$, and denote the result by $X_{g}(n)$. (The simple closed curve $a_{1}$ is depicted in Figure 4) Then by Lemma 2.3 the 4 -manifold $X_{g}(n)$ comes with a Lefschetz fibration $X_{g}(n) \rightarrow S^{2}$ of global monodromy $W_{g}(n)=W_{g} W_{g}^{t_{a_{1}}^{n}}=1$. (Here $W_{g}^{t_{a_{1}}^{n}}$ means the conjugate of $W_{g}$ by $t_{a_{1}}^{n}$.) Notice that by Remark 2.4. $W_{g}^{t_{a_{1}}^{n}}$ and hence $W_{g}(n)$ are products of right-handed Dehn twists.

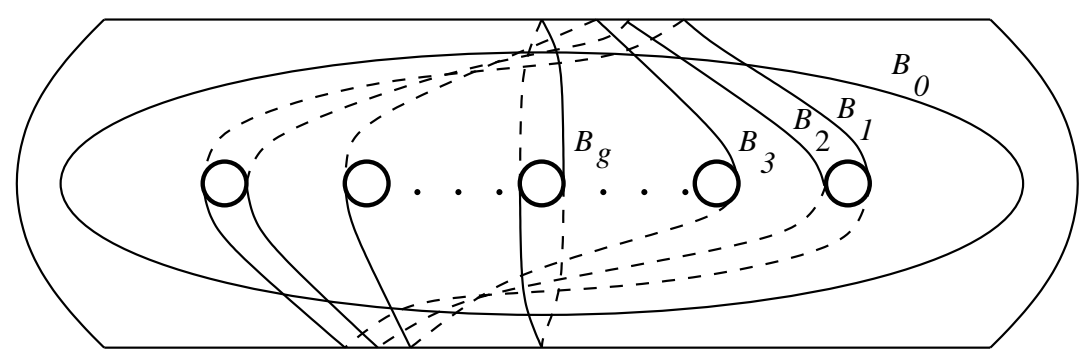

FIGURE 1.

It is routine to check that the curves $c_{1}, b_{1}, c_{2}, b_{2}, \cdots, c_{g}, b_{g}$ and $a_{2}$ (depicted in Figure 4) are fixed by the product $\left(t_{B_{0}} t_{B_{1}} t_{B_{2}} \cdots t_{B_{g}} t_{a}^{2} t_{b}^{2}\right)^{2}$ on the surface with one puncture. This shows that $\left(t_{B_{0}} t_{B_{1}} t_{B_{2}} \cdots t_{B_{g}} t_{a}^{2} t_{b}^{2}\right)^{2}=1$ in $\Gamma_{g, 1}$. Next we determine the element $W_{g}$ considered in $\Gamma_{g}^{1}$. It is known that $\operatorname{ker} \varphi=\left\{\Delta_{g}^{n}\right\} \cong \mathbb{Z}$ for the natural homomorphism $\varphi: \Gamma_{g}^{1} \rightarrow \Gamma_{g, 1}$ collapsing a boundary circle to a marked point. (Here $\Delta_{g}$ denotes the Dehn twist along a curve parallel to the boundary circle.) Hence $W_{g}=\Delta_{g}^{n}$ for some $n \in \mathbb{Z}$ follows from the above discussion.

Lemma 2.5. $\left(t_{B_{0}} t_{B_{1}} t_{B_{2}} \cdots t_{B_{g}} t_{a}^{2} t_{b}^{2}\right)^{2}=\Delta_{g}$ in $\Gamma_{g}^{1}$. 
Proof. We depicted $t_{b}^{2} t_{B_{0}} t_{B_{1}} t_{B_{2}} \cdots t_{B_{g}} t_{a}^{2} t_{b}^{2}(\tau)$ and $t_{B_{g-1}}^{-1} t_{B_{g-2}}^{-1} \cdots t_{B_{0}}^{-1} \Delta_{g}(\tau)$ in Figures 2 and 3, respectively, where $\tau$ is shown in Figure 4 One can use induction to obtain these figures. For example, the $W$-shaped part in the middle of Figure 3 is obtained by moving the legs of $W$ from outside to inside the holes when we apply $t_{B_{i}}^{-1} t_{B_{i-1}}^{-1}$. Now it is easy to see that

$$
t_{a}^{2} t_{b}^{2} t_{B_{0}} t_{B_{1}} t_{B_{2}} \cdots t_{B_{g}} t_{a}^{2} t_{b}^{2}(\tau)=t_{B_{g}}^{-1} t_{B_{g-1}}^{-1} \cdots t_{B_{0}}^{-1} \Delta_{g}(\tau),
$$

which proves the lemma.

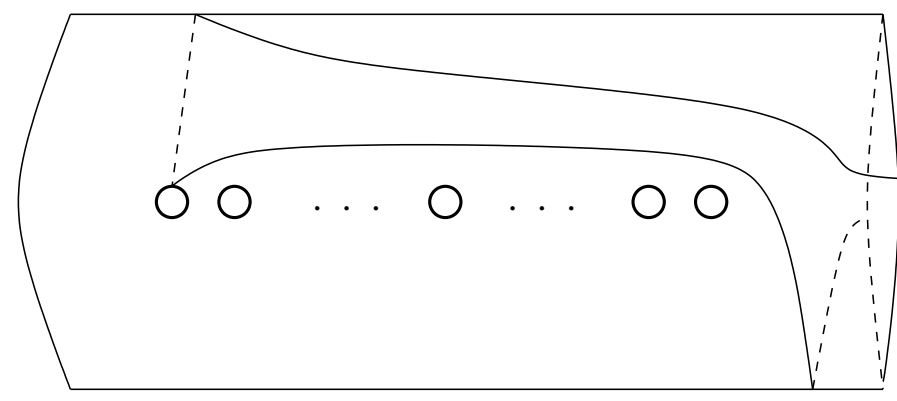

FIGURE 2. $t_{b}^{2} t_{B_{0}} t_{B_{1}} t_{B_{2}} \cdots t_{B_{g}} t_{a}^{2} t_{b}^{2}(\tau)$

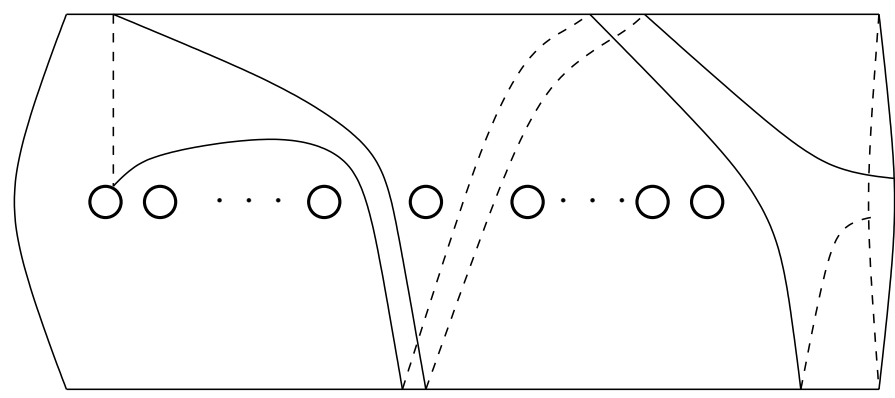

FiguRE $3 . t_{B_{g-1}}^{-1} t_{B_{g-2}}^{-1} \cdots t_{B_{0}}^{-1} \Delta_{g}(\tau)$

Corollary 2.6. The Lefschetz fibration $X_{g}(n) \rightarrow S^{2}$ admits a section $\sigma_{g}(n)$ with self-intersection number -2 .

Proof. We saw that the relator $W_{g}$ admits a lift under the obvious map $\Gamma_{g, 1} \rightarrow \Gamma_{g}$ defined by forgetting the marked point. Once such a lift is fixed, it gives a section $\sigma_{g}$ of the Lefschetz fibration $X_{g} \rightarrow S^{2}$. Similarly we get a section $\sigma_{g}(n)$ of $X_{g}(n) \rightarrow S^{2}$. Lemma 2.5proved that $\Delta_{g}=W_{g}$ and thus $\Delta_{g}^{2}=W_{g}(n)$ in $\Gamma_{g}^{1}$, since $t_{a_{1}}^{n} \Delta_{g} t_{a_{1}}^{-n}=\Delta_{g}$. It is known (see [18]) that if for the relator $w$ we have $w=\Delta_{g}^{k}$ in $\Gamma_{g}^{1}$, then the Lefschetz fibration $X \rightarrow S^{2}$ given by $w$ admits a section of square $-k$. This observation concludes the proof.

Since our Lefschetz fibrations admit sections, Lemma 2.2 can be applied to compute their first homology groups: 
Lemma 2.7. The first homology group $H_{1}\left(X_{g}(n) ; \mathbb{Z}\right)$ of $X_{g}(n)$ is isomorphic to $\mathbb{Z}^{g-2} \oplus \mathbb{Z}_{n}$.

Proof. In the following we will denote the homology classes of curves by the same letters as we denote the curves. Let $a_{1}, b_{1}, a_{2}, b_{2}, \cdots, a_{g}, b_{g}$ denote the standard generators of the first homology group of the fiber, as depicted in Figure 4 It is easy to see that $B_{i}^{t_{a_{1}}^{n}}=B_{i}$ for $i \geq 2$, and for an appropriate choice of orientation on $B_{i}$, in homology we have

$$
\begin{gathered}
B_{g}=a+b_{r+1}+b, \quad B_{0}=b_{1}+b_{2}+\cdots+b_{g} \\
B_{i}^{t_{a_{1}}^{n}}=B_{i}+n a_{1} \quad \text { for } i=0,1 \\
B_{2 i-1}=b_{i}+B_{2 i}+b_{g-(i-1)}
\end{gathered}
$$

and

$$
B_{2 i}=a_{i}-a_{i+1}+B_{2 i+1}+a_{g-(i-1)}-a_{g-i}
$$

for $i=1, \cdots, r$, where $g=2 r+1$. Thus by Lemmas 2.2 and 2.3 the homology $H_{1}\left(X_{g}(n) ; \mathbb{Z}\right)$ is an (abelian) group generated by $a_{1}, b_{1}, a_{2}, b_{2}, \cdots, a_{g}, b_{g}$ with relations

$$
a_{r+1}=b_{r+1}=0, \quad a_{i}+a_{g-(i-1)}=0, \quad b_{i}+b_{g-(i-1)}=0, \quad n a_{1}=0 .
$$

Now Lemma 2.2 implies the result.

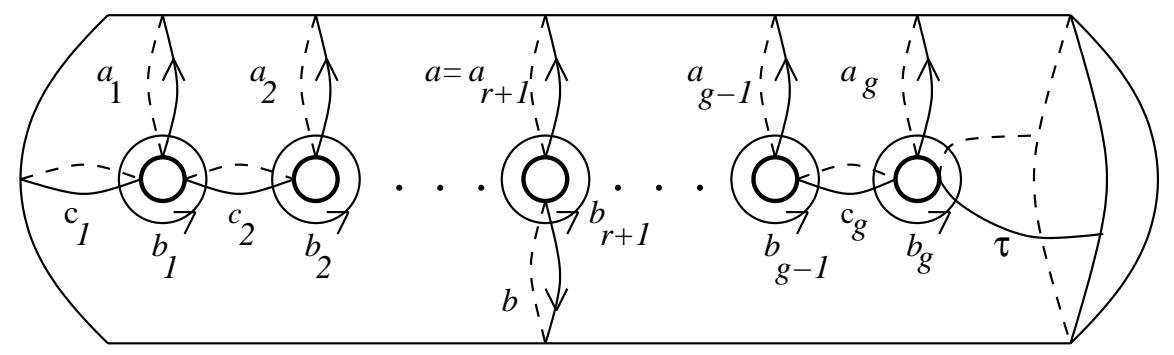

FIGURE 4.

In fact, for any given integer $0 \leq k \leq g-2$ and $n \geq 1$, we can construct a Lefschetz fibration over $S^{2}$ with first homology group isomorphic to $\mathbb{Z}^{k} \oplus \mathbb{Z}_{n}$, by taking twisted fiber sums of more copies of $X_{g}$. We could use any of these fibrations in the rest of the paper as well.

Remark 2.8. If $g=2 r \geq 2$ is even, similar results can be obtained for fibrations defined by the relator $W_{g}=\left(t_{B_{0}} t_{B_{1}} t_{B_{2}} \cdots t_{B_{g}} t_{c}\right)^{2}$ (see Figure 5). In the exact same manner we can construct $X_{g}$ and the twisted fiber sums $X_{g}(n) \rightarrow S^{2}$ with sections of square -2 . The homology computation applies without essential change, and provides $H_{1}\left(X_{g}(n) ; \mathbb{Z}\right)=\mathbb{Z}^{g-2} \oplus \mathbb{Z}_{n}$. (The observation preceding this remark also has its natural extension to the even $g$ case.) The only notable difference is that for $g$ even the relator $W_{g}$ contains homologically trivial vanishing cycles as well cf. the curve denoted by $c$ in Figure 5 . 


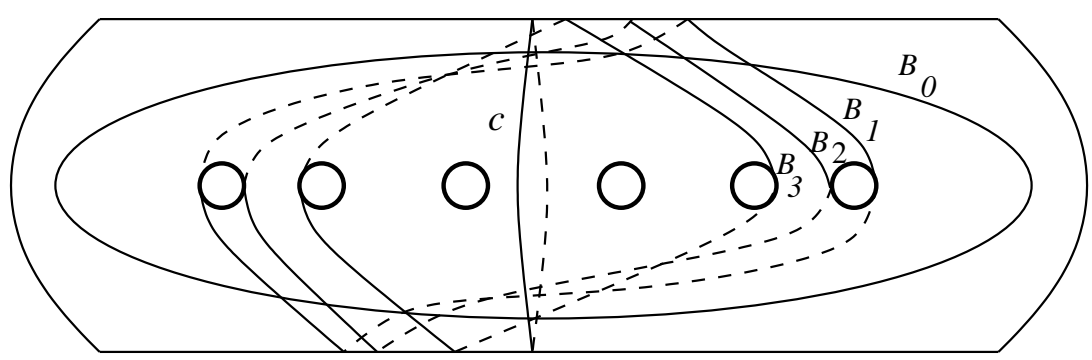

FIGURE 5. Vanishing cycles in the even $g$ case

\section{Stein structures on Lefschetz Fibrations With Boundary}

Definition 3.1. Let $X$ be a compact 4-manifold with boundary. The map $\pi: X \rightarrow$ $B$ is a Lefschetz fibration if $\pi$ satisfies the requirements of Definition 2.1 with the possible exemption of allowing $\pi^{-1}(\partial B) \neq \partial X$, and assuming that $\pi$ is a fiber bundle map away from the singular fibers. (Notice that this definition allows the fiber $\pi^{-1}(t)$ to have a boundary.) In the following, LFB (Lefschetz fibration with bounded fibers) will denote a relatively minimal Lefschetz fibration over $D^{2}$ whose generic fiber is a surface with nonempty boundary.

Theorem 3.2 ([3], [7], [14]). Let $f: X \rightarrow S^{2}$ be a Lefschetz fibration with a section $\sigma$ and let $\Sigma$ denote a regular fiber of this fibration. Then $S=X-\operatorname{int} \nu(\Sigma \cup \sigma)$ is a Stein filling of its boundary equipped with the induced (tight) contact structure, where $\nu(\Sigma \cup \sigma)$ denotes a regular neighborhood of $\Sigma \cup \sigma$ in $X$.

We only sketch the proof of the above theorem, since it was already proved in [14], 3] (with the extra assumption of having only homologically essential vanishing cycles in the fibration), and also appeared in [7].

Proof of Theorem 3.2 (sketch). When we remove int $\nu(\Sigma \cup \sigma)$ from $X$, it is clear that we get an LFB. Thus $S=X-\operatorname{int} \nu(\Sigma \cup \sigma)$ admits a standard handlebody decomposition: It is obtained by a sequence of steps of attaching 2-handles $S_{0}=$ $D^{2} \times F \rightsquigarrow S_{1} \rightsquigarrow S_{2} \cdots \rightsquigarrow S_{n}=S$, where each $S_{i-1}$ is an LFB and $S_{i}$ is obtained from $S_{i-1}$ by attaching a 2 -handle along a simple closed curve $\gamma$ lying on a fiber $\Sigma^{\prime} \subset \partial S_{i-1}$. Furthermore, this handle is attached to $\gamma$ with the framing $k-1$, where $k$ is the framing induced from the surface $\Sigma^{\prime}$ (see the text after Definition 2.1). Inductively we assume that $S_{i-1}$ has a Stein structure, with a convex fiber $\Sigma^{\prime} \subset$ $\partial S_{i-1}$. By the "Legendrian realization principle" of [1] (pp. 323-325) applied to the homologically essential vanishing cycle $\gamma$, after an isotopy of $\left(\Sigma^{\prime}, \gamma\right), \gamma$ becomes a Legendrian curve and $k$ can be taken to be its Thurston-Bennequin framing. If $\gamma$ is homologically inessential, the above principle does not apply verbatim. In this case choose a homologically essential simple closed curve in the component of the complement of $\gamma$ that is disjoint from $\partial \Sigma^{\prime}$. Isotope it to Legendrian position (by the above argument) and, using the local model of the contact structure along this Legendrian $\operatorname{knot} \delta$, isotope $\Sigma^{\prime}$ to introduce new dividing curves parallel to $\delta$. Now the Legendrian realization principle applies to $\gamma$, since after this modification (called a fold) each component of its complement intersects the set of dividing curves nontrivially. (For the relevant definitions see [6], [11].) Now a celebrated 
result of Eliashberg [5], [10] provides an extension of the Stein structure of $S_{i-1}$ to $S_{i}$

Remark 3.3. Notice that if $g$ is odd, then all vanishing cycles are homologically essential; hence in this case no fold is necessary. In the even $g$ case our examples contain homologically inessential vanishing cycles ( $c$ on Figure 5); hence application of folds is necessary.

Definition 3.4. Let $M$ be a closed 3-manifold. An open book decomposition of $M$ is a pair $(K, \pi)$ consisting of a (fibered) link $K$, called the binding, and a fibration $\pi: M-K \rightarrow S^{1}$ such that each fiber is a Seifert surface for $K$. (The fibers are also called the pages of the open book.) Let $\Sigma^{\prime}$ denote a surface with boundary. The closed 3-manifold

$$
M=\left(\left(\Sigma^{\prime} \times I\right) /(h(x), 0) \sim(x, 1)\right) \cup_{\partial}\left(\partial \Sigma^{\prime} \times D^{2}\right)
$$

is canonically decomposed as an open book with binding $\partial \Sigma^{\prime}$, page $\Sigma^{\prime}$ and monodromy $h$.

It is easy to see that the boundary of an LFB has a canonical open book decomposition induced from the fibration. The pages are the fibers in the boundary of the LFB, while the monodromy of the open book is just the total monodromy of the LFB along $S^{1}=\partial D^{2}$. The binding is simply the boundary of the central fiber $\pi^{-1}(0)$.

We now turn our attention to contact structures on 3-manifolds. In [21], Thurston and Winkelnkemper constructed a contact structure associated to a given open book decomposition of a closed, orientable 3-manifold. Recently Giroux [9] refined this construction by showing that an open book $(K, \pi)$ supports a unique (up to isotopy) compatible contact structure $\xi$, where a contact structure $\xi$ is called compatible with an open book $(K, \pi)$ if there is a contact 1-form $\alpha$ for $\xi$ such that $d \alpha$ is a volume form on each fiber of $\pi$ and the binding $K$ is a transverse link in $(M, \xi)$ (oriented as the boundary of a page). In fact, Giroux established a bijection between the set of isotopy classes of contact structures on a closed 3-manifold $M$ and open books supported by $M$; here two open books are considered to be equivalent if they can be joined by a sequence of positive stabilization/destabilization. In addition, Giroux proved that $\xi$ is Stein fillable if the monodromy of the compatible open book decomposition can be expressed as a product of right-handed Dehn twists. Notice that the boundary of an LFB admits two contact structures - one induced by the Stein structure of the LFB, and the other given by the open book through the total monodromy of the Lefschetz fibration. The above result of Giroux [9] essentially states that these two structures are isotopic. (A slightly different proof of the same statement - resting on results of Torisu [22] - has been given by Gay [8].)

Proposition 3.5 (7], 8], 9]). The contact structure on the boundary of an LFB (induced from the Stein structure) is isotopic to the contact structure associated to the boundary of this LFB considered as an open book. In particular, any two LFB's bounding the same positive open book decomposition are Stein fillings of the same contact structure.

Remark 3.6. In proving this proposition one first checks it for the trivial LFB with no critical points (hence with total monodromy equal to id). In this case the Lefschetz fibration is built using 1-handles only. Then an induction on the number 
of 2-handles together with the observation that the handle attachment (which is surgery on the 3-manifold level) yields an open book compatible with the new contact structure proves the result. (This last observation only involves a local check along a page of the open book.)

Now we apply the above discussion to the Lefschetz fibrations $X_{g}(n) \rightarrow S^{2}$ constructed in Section 2, Let $\Sigma_{g}(n)$ denote a regular fiber of the Lefschetz fibration $X_{g}(n) \rightarrow S^{2}$ with section $\sigma_{g}(n)$. Let $S_{g}(n)$ denote $X_{g}(n)-\operatorname{int} \nu\left(\Sigma_{g}(n) \cup \sigma_{g}(n)\right)$.

Lemma 3.7. The first homology group $H_{1}\left(S_{g}(n) ; \mathbb{Z}\right)$ of $S_{g}(n)$ is isomorphic to $H_{1}\left(X_{g}(n) ; \mathbb{Z}\right)=\mathbb{Z}^{g-2} \oplus \mathbb{Z}_{n}$.

Proof. $H_{1}\left(X_{g}(n) ; \mathbb{Z}\right) \cong H_{1}\left(S_{g}(n) ; \mathbb{Z}\right)$ follows from the observation that the normal circle to the fiber is homologous to a multiple of the normal circle to the section (shown by a push-off of the section), which bounds a punctured fiber, hence is zero in $H_{1}\left(S_{g}(n) ; \mathbb{Z}\right)$.

Proof of of Theorem 1.1. Let $M_{g}(n)$ denote the boundary of $S_{g}(n)$ with the contact structure $\xi_{g}(n)$ induced by the open book provided by $S_{g}(n)$ as an LFB. Notice that $M_{g}(n)=-\partial \nu(\Sigma \cup \sigma)$; hence its diffeomorphism type does not depend on $n$. Moreover, the total monodromy of $S_{g}(n)$ is equal to $\Delta_{g}^{2} \in \Gamma_{g}^{1}$; hence the open book, and therefore the contact structure $\xi_{g}(n)$, are independent of $n$. Let $\left(M_{g}, \xi_{g}\right)$ denote $\left(M_{g}(n), \xi_{g}(n)\right)$. According to Proposition 3.5 the Stein structures on $S_{g}(n)$ $(n=1,2, \ldots)$ all provide Stein fillings of $\left(M_{g}, \xi_{g}\right)$, and using Lemma 3.7we conclude that these are pairwise non-diffeomorphic fillings.

Since $H_{1}\left(M_{g} ; \mathbb{Z}\right)=\mathbb{Z}^{2 g}$, we also see that $M_{g}$ is diffeomorphic to $M_{g^{\prime}}$ if and only if $g=g^{\prime}$; therefore, the proof of Theorem 1.1 is complete. Since $\nu(\Sigma \cup \sigma)$ is just the plumbing of the disk bundle over a genus- $g$ surface with Euler number 0 and the disk bundle over a sphere with Euler number -2, we get that $M_{g}$ (which is $\partial \nu(\Sigma \cup \sigma)$ with the opposite orientation) is the boundary of a similar plumbing (now with Euler number 2), as stated in Section 1 .

Remark 3.8. The above construction just gave different factorizations of the mapping class $\Delta_{g}^{2} \in \Gamma_{g}^{1}$ into products of right-handed Dehn twists. In fact, any such factorization of an element $h \in \Gamma_{g}^{r}(r>0)$ provides a Stein filling of the contact structure induced by $h$, and the topological argument (computing the first homology) helped us to distinguish the various factorizations of $\Delta_{g}^{2}$. In the light of this observation we can weaken Conjecture 1.2 to

Conjecture 3.9. For $h \in \Gamma_{g}^{r}(r>0)$ there exists a constant $C_{h}$ such that if $h=t_{1} \cdots t_{n}$ with $t_{i}$ right-handed Dehn twists, then $n \leq C_{h}$.

The following observation can serve as evidence for Conjecture 3.9.

Lemma 3.10. For $g$ odd the Euler characteristic $\chi\left(S_{g}(n)\right)$ and the signature $\sigma\left(S_{g}(n)\right)$ of $S_{g}(n)$ are equal to 11 and -16 , respectively.

Proof. For $g$ odd the Stein filling $S_{g}(n)$ admits an LFB with $2 g+10$ singular fibers. Thus,

$$
\chi\left(S_{g}(n)\right)=\chi\left(D^{2}\right) \chi(\text { fiber })+2 g+10=2-2 g-1+2 g+10=11 .
$$

The signature $\sigma\left(X_{g}(n)\right)=-16$, since $\sigma\left(X_{g}\right)=-8[12$ and the signature is additive under fiber sum. This implies that $\sigma\left(S_{g}(n)\right)=-16$, since we remove a piece with zero signature to get $S_{g}(n)$ from $X_{g}(n)$. 
(For $g$ even a similar computation gives $\chi\left(S_{g}(n)\right)=5$ and $\sigma\left(S_{g}(n)\right)=-8$.) Notice that the length of the factorization $h=t_{1} \cdots t_{n}$ into right-handed Dehn twists is intimately related to the Euler characteristic of the Stein filling induced by $t_{1} \cdots t_{n}$.

\section{ACKNOWLEDGMENT}

The authors would like to thank Selman Akbulut, Mustafa Korkmaz and John Etnyre for many helpful and inspiring discussions. The second author was partially supported by OTKAT34885.

\section{REFERENCES}

[1] B. Aebischer, M. Borer, M. Kälin, Ch. Leuenberger, and H. M. Reimann, Symplectic geometry, Progress in Math. 124, Birkhäuser Verlag, Basel, 1994. MR 96a:58082

[2] S. Akbulut and B. Ozbagci, Lefschetz fibrations on compact Stein surfaces, Geom. Topol. 5 (2001), 319-334; errata, ibid., 939-945. MR 2003a:57055, MR 2003b:57043

[3] S. Akbulut and B. Ozbagci, On the topology of compact Stein surfaces, Internat. Math. Research Notices 15 (2002), 769-782. MR 2003a:57049

[4] Y. Eliashberg, Filling by holomorphic disks and its applications, Geometry of LowDimensional Manifolds: 2, Proc. Durham Sympos. 1989, London Math. Soc. Lecture Notes, 151, Cambridge Univ. Press, 1990, 45-67. MR 93g:53060

[5] Y. Eliashberg, Topological characterization of Stein manifolds of dimension $>2$, Internat. J. of Math. 1 (1990), 29-46. MR 91k:32012

[6] J. Etnyre, Introductory lectures on contact geometry, preprint (arXiv: math.SG/0111118).

[7] J. Etnyre and K. Honda, On symplectic cobordisms, Math. Ann. 323 (2002), 31-39. (arXiv: math.SG/0101145). MR 2003c:57026

[8] D. Gay, Explicit concave fillings of contact three-manifolds, Math. Proc. Cambridge Philos. Soc. 133 (2002), 431-441. (arXiv:math.GT/0104059). MR 2003i:57041

[9] E. Giroux, Lecture at Oberwolfach, 2001. (See also: http://www.math.uga.edu/ topology/ giroux.pdf)

[10] R. Gompf and A. Stipsicz, 4-manifolds and Kirby calculus, Graduate Studies in Math., Vol. 20, Amer. Math. Soc., Providence, RI, 1999. MR 2000h:57038

[11] K. Honda, On the classification of tight contact structures I, Geom. Topol. 4 (2000), 309-368. MR 2001i:53148

[12] M. Korkmaz, Noncomplex smooth 4-manifolds with Lefschetz fibrations, Internat. Math. Research Notices 2001, 115-128. MR 2001m:57036

[13] P. Lisca, On lens spaces and their symplectic fillings, preprint (arXiv: math.SG/0203006).

[14] A. Loi and R. Piergallini, Compact Stein surfaces with boundary as branched covers of $B^{4}$, Invent. Math. 143 (2001), 325-348. MR 2002c:53139

[15] D. McDuff, The structure of rational and ruled symplectic 4-manifolds, Journal of the Amer. Math. Soc. 3 (1990), 679-712; erratum, ibid. 5 (1992), 987-988. MR 91k:58042 MR 93k:58098

[16] H. Ohta and K. Ono Simple singularities and symplectic fillings, preprint 2002.

[17] H. Ohta and K. Ono Symplectic fillings of the link of simple elliptic singularities, preprint 2002.

[18] I. Smith, Geometric monodromy and the hyperbolic disc, Quarterly J. Math. Oxford Ser. (2) 52 (2001), 217-228. MR 2002c:57046

[19] I. Smith, Torus fibrations on symplectic four-manifolds, Turkish J. Math. 25 (2001), 69-95. MR 2002c: 57047

[20] A. I. Stipsicz, Gauge theory and Stein fillings of certain 3-manifolds, Turkish J. Math. 26 (2002), 115-130. MR 2003b:57038 
[21] W. P. Thurston and H. Winkelnkemper, On the existence of contact forms, Proc. Amer. Math. Soc. 52 (1975), 345-347. MR 51:11561.

[22] I. Torisu, Convex contact structures and fibered links in 3-manifolds, Internat. Math. Research Notices 2000, 441-454. MR 2001i:57039

College of Arts and Sciences, Koc University, Rumelifeneri Yolu 34450, Sariyer, ISTANBUL, TURKEY

E-mail address: bozbagci@ku.edu.tr

A. Rényi Institute of Mathematics, Hungarian Academy of Sciences, Budapest, Hungary and Department of Mathematics, Princeton University, Princeton, New Jersey 08544

E-mail address: stipsicz@math-inst.hu

E-mail address: stipsicz@math.princeton.edu 